\title{
Method Transfer and Full Method Validation, Partial Validation in cGMP Environment
}

\author{
Sai Raghuveer Chava1*, Jingbo Liu² and Sajid Bashir² \\ ${ }^{1}$ Analytical Development, Emergent BioSolutions, Gaithersburg, MD, USA \\ ${ }^{2}$ Department of Chemistry, Texas A\&M University-Kingsville, USA
}

*Corresponding author: Sai Raghuveer Chava, Analytical Development, Emergent

BioSolutions, 300 Professional Drive, Gaithersburg, Maryland 20879 USA, Tel: 240-631-3200; Email: ChavaS@ebsi.com

\section{Review Article \\ Volume 2 Issue 2}

Received Date: December 04, 2019

Published Date: December 13, 2019

DOI: $10.23880 /$ aabsc- 16000143

\section{Abstract}

This paper describes the current method transfer and method validation process following FDA guidance and ICH guidelines in a cGMP environment. The analytical method validation and transfer have been playing a key role in Phase 1 to Phase 3 projects. This paper discusses the method validation and method transfer based on the phase of relevance in the GMP environment. It also describes the number of validation or qualification steps required for the method transfer. When the analytical methods are utilized to generate results about the characteristics of drug-related samples it is essential that the results are trustworthy. They may be utilized as the basis for decisions relating to administering the drug to patients. Analytical method validation required during drug development and manufacturing and these analytical methods is fit for their intended purpose. To comply with the requirements of GMP pharmaceutical industries should have an overall validation policy that documents how validation will be performed. The purpose of this validation is to show that processes involved in the development and manufacture of the drug, production, and analytical testing can be performed in an effective and reproducible manner. This review article provides guidance on how to perform validation characteristics for the analytical method which are utilized in pharmaceutical analysis.

Keywords: Validation Methods; Analytical Methodology; cGMP; ICH Guidelines

\section{Introduction}

The method validation is a process by which the testing laboratory performs a range of different steps following the method intended application to meet the ICH Q2 (R1). The method validation following ICH Q2(R1) typically recommends evaluating the following analytical characteristics of a method: Accuracy, Precision, Specificity, Detection Limit, Quantitation Limit, Linearity, Range and Robustness. A limited number of analytical characteristics will be required for validation based on the type of method and its use in the project [1]. 


\section{Validation}

Method Transfer refers to the transfer of previously validated or qualified, a non-compendial method to different laboratories or sites for the testing same type of sample. Method Transfer can be performed using different strategies. The "sending" laboratory (the laboratory from which the method is being transferred) may perform the full validation and partial validation may be performed on the "receiving" laboratory (the laboratory to which a method being transferred) to show the feasibility and correlation between the results. Both "sending" and "receiving" laboratory may have different strategies for the method transfer, but the final goal is to qualify or validate the method appropriately following ICH guidelines [2].

\section{Method Verification of Compendial Methods}

In the USP, method verification is defined as an evaluation of whether a Compendial method is suitable for the intended use of the raw material or product analysis as required by a manufacturer. Thus, method verification is specific to the laboratory executing the test and to the sample type being tested. Specific details regarding method verification may be found in USP General Chapter <1226> entitled "Verification of Compendial Procedures" [3] (Figure 1).

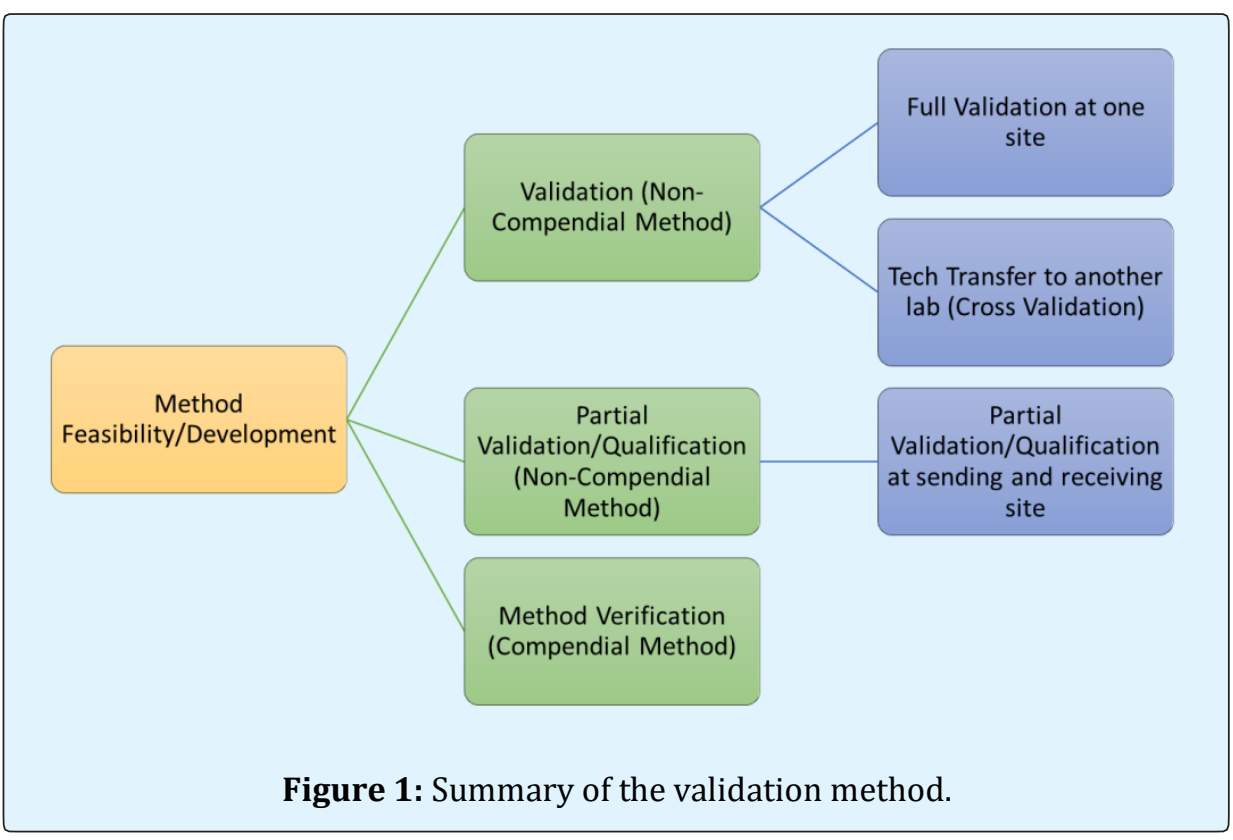

\section{Method Validation}

Analytical method validation is highly dependent on the understanding of the following terms before validation:

1. Spiking: Spiking of a known analyte is very low to be injected in a method like HPLC because the peak contained in the chromatogram may be very small, which may not provide very accurate reading as desired.

2. Average/Mean: Average result $\bar{x}$, is calculated by summing the individual results and dividing the sum by the number $(\mathrm{n})$ of individual values.
The average result, $\bar{x}=\frac{x 1+x 2+x 3+x 4+\ldots \ldots}{n}$

3. Standard Deviation: The standard deviation is a measure to check the accuracy of the average value. It is a measure of the random error which we cannot control. It is calculated as below.

$$
\text { Standard deviation, } S=\sqrt{\frac{\left(x_{1}-\bar{x}\right)^{2}+\left(x_{2}-\bar{x}\right)^{2}+\cdots}{n-1}}
$$

4. Relative Standard deviation: The relative standard deviation (RSD) is expressed in percentage and obtained by multiplying the standard deviation by 100 and dividing the result by average.

$$
\text { Relative standard deviation, } \mathrm{RSD}=100 \frac{\mathrm{s}}{\bar{x}}
$$


Validation is a protocol guided demonstration of the key attributes of a method. The listed parameters are provided below. The characteristics of the analytical method should be within the prescribed limit and defined standards to confirm its accuracy and authenticity.

- Accuracy: Accuracy is well-defined as a measure of the trueness of the analytical method. It is the closeness of test results to the true value. Accuracy is generally determined from the percent recovery of a known amount of analyte added to a blank or a sample.

- Precision: Precision is a measure of the degree of repeatability and reproducibility of the analytical method under normal operating circumstances. The precision of an analytical method is the degree of agreement among individual tests when the procedure is applied to multiple portions of a homogeneous sample. The precision is expressed as the standard deviation (SD) and relative standard deviation (RSD) or the coefficient of variation (CV).

\section{- Precision}

a) Repeatability: Repeatability is to evaluate the variation experienced by a single analyst under the same operating condition over a short interval of time.

b) System Suitability: A set of empirically determined parameters that are set to assure that the entire method is functional prior to acceptance of any given set of test results.

c) Intermediate Precision: Intermediate precision evaluates the variation in a laboratory on different days, instruments, and analysts

- Specificity: Specificity determines the presence or absence of interference from complex mixtures. It is determined by comparing the analyte in the presence of impurities, degradants, matrix, and known excipients.

- Linearity: Linearity is the ability of an analytical test method to provide test results that are directly proportional to the concentration of an analyte in samples within a given range.

- Limit of Quantitation (LOQ): Limit of quantitation is the lowest amount of analyte in a sample which can be quantitatively determined with suitable precision and accuracy.

- Limit of Detection (LOD): Limit of detection is the lowest amount of analyte in a sample that can be reliably detected but not necessarily quantitated as an exact amount. Detection limit or limit of detection (LOD) can be determined from signal-to-noise ratio $(\mathrm{S} / \mathrm{N})$ of a sample of known concentration and/or the standard deviation of the response and the slope of the calibration curve.

\section{Conclusion}

An overview of the fundamental principles, design criteria, outline of process is given within the framework of risk management solution designed to facilitate risk-based qualification, process validation, and change control activities within GMP and Pharmaceutical regulatory compliance environments as well as the variance used therein.

\section{Acknowledgment}

The authors are grateful to the National Science Foundation (NSF) Centers of Research Excellence in Science and Technology (NSF CREST, HRD-0734850, and CBET MRI-0821370) at the Texas A\&M UniversityKingsville. The Robert C Welch (AC0006) Departmental Grant is also duly acknowledged. In addition, the supervisory staff at Emergent BioSolutions for their advice and assistance in the preparation of this report. Note: S. Chava conceived the project, S. Bashir edited the manuscript and J. Liu checked the figure.

\section{References}

1. O'Donnell K, Greene A (2006) A risk management solution designed to facilitate risk-based qualification, validation, and change control activities within GMP and Pharmaceutical regulatory compliance environments in the EU, Part I: fundamental principles, design criteria, an outline of the process. Journal of GXP Compliance 10(4): 12-26.

2. Graham AT, Lee CJ, Powell LH, Smallshaw HR, Ulman ST (2015) Implementation of ICH Q3D elemental impurities guideline: challenges and opportunities. Pharmaceutical Technology 39(3): 1-3.

3. Ermer J, Miller JHM (2006) Method validation in pharmaceutical analysis: A guide to best practice [1 $1^{\text {st }}$ Edition], John Wiley \& Sons, New York, USA, pp: 1140 . 